\section{"NOTHING OF THE ORIGIN AND DESTINY OF CATS":} THE REMAINCER OF THE LOGOS

\author{
John M. Rose \\ Goucher Collega
}

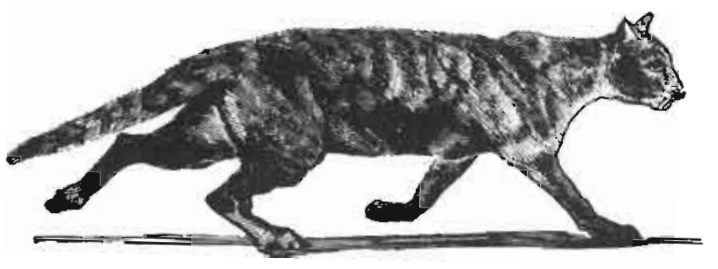

\section{Editors' Note: This paper was presented at the Central Division Meeting of the Society for the Study of Ethics and Animals, held in Chicago, Illinois, April, 1989.}

Henry David Thoreau once posed a question which now challenges us as philosophers who would take up the issue of ethics and animals: "What sort of philosophers are we, who know absolutely nothing about the origin and destiny of cats?" This question is a spur to us. Even if we object that the question requires qualification, that "absolutely nothing" is too strong, that philosophers have indeed considered the question of the origins of animals, that we know something...even if we object that Thoreau died too young (1862) to fully comprehend Darwin's arigin of the Species (1859)...the question provokes us afresh. Thoreau's question both cites and sites a failure of philosophy and philosophers: because we know nothing of the origin and destiny of cats we fail as philosophers. The question, further, situates the failure in one endeavor: knowing cats. A shift, a

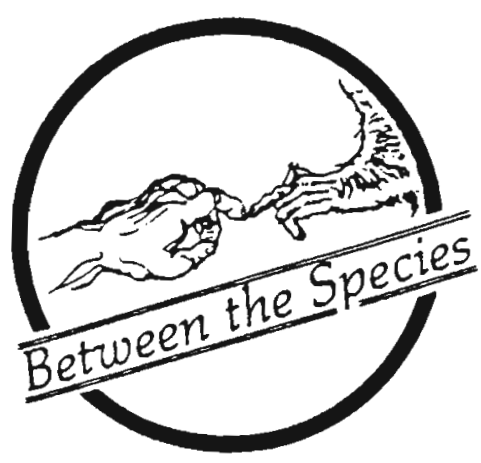

movement away from the goal of self-knowledge by which philosophers have traditionally judged themselves takes place in Thoreau's question. Even if we consider the situation of the question unfair or rhetorically whimsical, it remains a spur. And notice that this last dichotomy is a movement in the question itself: the question is an absolute condemnation and absolutely whimsical. To engage in a reflection on ethics and animals, I must know something of their origins and destinies. Specifically, I must begin with cats.

As a philosopher, I first of all wonder how I am to place myself in the situation of this question... whimsically or seriously. And being a philosopher, I of course question the question, an act which opens up a plethora of questions which expand in ever-increasing generality and then contract: must I become a biologist before I can undertake an ethical consideration of animals? Can knowledge of origins and destinies be necessary? By what methods, hypotheses, and assumptions am 1 to proceed? Why cats?

in the face of such questions as these, I propose a clarification of the situation in which Thoreau's

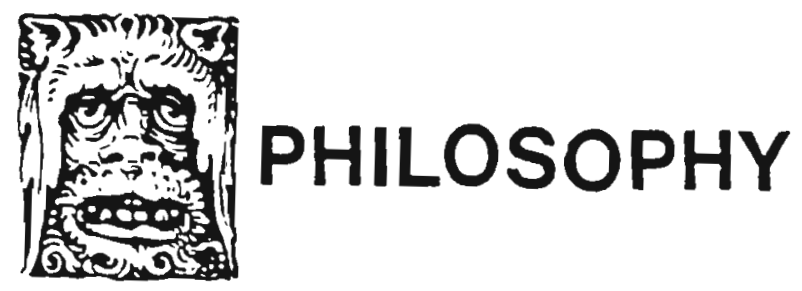


question places us as philosophers who approach the issue of ethics and animals. I propose that Thoreau's question aboul philosophy and the origin and destiny of cats places us within the framework of a central motif which philosophy adopts when it takes up questions about animals. This central motif is that of production, and it entails the consideration of all the leitmotifs that are commensurate with it: necessity of design, the dominance of the end or goal or that for the sake of which it is, orchestrated behavior, and most fundamentally the origin of all of these, the logos. In response to Thoreau's question, I ask "What sort of philosophy is it that asks questions about origins and destinies of animals?"

To achieve a clarification of the philosophical situation in which this question is asked, I propose an examination of two texts: Aristotle's De Partibus Animalium and Heidegger's Sein und Zeit. Specifically, I undertake an exegesis of their respective discussions of the method for inquiring about the origins and destinies of animals. I show that in the matter of animals the method of both philosophers is structured and guided by the metaphor of human production. I further argue that in the thought of both philosophers the metaphor of productivity dominates their respective notions of logos. I conclude by arguing that, if we see that Thoreau's question is itself a reflection of this dominant metaphor, it becomes important for a consideration of ethics and animals to ask, "What does the dominance of the metaphor of human productivity imply for the ethical treatment of animals? What remains of the logos that is not dominated by the metaphor of human productivity?"

Provoked by Thoreau's question, I now turn to the methodological consideration in Aristotle's treatise on The Parts of Animals.

When Aristotie gives consideration to the question of origin and destiny of animals, he turns the question towards the larger issue of the causes in the generation of the works of nature. There are two such causes: the final cause and the motor cause. Of these, the former is the logos of the generated being. (My cal, Thomisina, is constantly on the lookout for a cat toy that is its own motor cause, so we shall leave the issue of efficient causality to her.) The logos provides us with the proximate origin of anything made or produced, be it artifact or animal:

There is the Cause for the sake of which the thing is formed, and the Cause to which the beginning of the motion is due. Therefore another point for us to decide is which of these two Causes stands first and which comes second. Clearly, the first which we call the "Final" Cause--that for the sake of which the thing is formed-since that is the logos of the thing--its rational ground, and the logos is always the beginning for products of Nature as well as for those of Art. $(639 \mathrm{~b} 113-17)^{\prime}$

Aristolle's argument stresses the univocal character of the final cause in the works of art and the works of nature. Both are products determined by their respective logoi, and the site of this determination is illustrated with the example given by Aristotle which follows this passage. To understand production in nature, Aristotle provides the analogy of the procedure of the builder and the physician. $(O r$ is it an example? This determination remains an issue which my interpretation must confront.) The builder and the physician start by forming a definite picture of the end or goal which they hold to as the reason and justification for each successive step. As the builder and physician pursue a goal which determines the best means, 50 also works of nature have a good end and final cause which they hold to in producing the successive stages that move towards their end. Aristotle states that the final cause is even more dominant in nature than in works of art and is the usual way in which most writers on this subject define the origin.

The final cause in works of nature is not aiways necessary, and, what necessity there is, is qualified by various degrees. Absolute necessity only applies purely to eternal phenomena. The materials for production and the order in which the steps are taken to produce something are also necessary. Further, each necessary condition demands the pre-existence or previous production of a particular antecedent. But, of course, the existence of a particular antecedent does not make it necessary that the 
particular product comes into existence. With the question of the origin of animals, we have found layers of sequenced production, none of which needs to result in a product but all of which are necessary if the product is to exist.

After a critique of Empedocles, Aristotle points out both the best and second-best procedures for investigating these questions about the origins of animals:

The fittest mode, then, of treatment is to say, a man has such and such parts, because the conception of a man includes their presence, and because they are necessary conditions of his existence, or, if we cannot quite say this, which would be best of all, then the next thing to it, namely, that it is either quite impossible for him to exist without them, or, at any rate, that it is better for him that they should be there, and their existence involves the existence of other antecedents. Thus we should say, because man is an animal with such and such characteristics, therefore is the process of his development necessarily such as it is; and therefore is it accomplished in such and such an order, this part being formed first, that next, and so on in succession; and after a like fashion should we explain the evolution of all other works of nature. $(640 a 33-640 b 4)^{2}$

The conception of man, or any other animal...the conception which includes the presence of the parts it is best for any animal to have...this conception is the final cause for that animal. We are thus brought back to the univocal character of the task of determining the origins of animals and other artifacts. The procedure for determining the logos of an artifact is the same as the procedure for determining the logos of any animal. Aristotle in fact states:

It is plain, then, that the teaching of the old physiologists is inadequate, and that the true method is to state what the definitive characters are that distinguish the animal as a whole; to explain what it is both in substance and in form, and to deal after the same fashion with its several organs; in fact, to proceed in exactly the same way as we should do, were we giving a complete description of a couch. (641a/4-18)

As everyone familiar with Aristotle knows, the doctrine of matter and form, or more specifically the notion of ensouled matter, allows him to separate the task of the natural philosopher in treating animals from the task of another philosopher describing the production of artifacts. Even with this separation, however, Aristotle still preserves the parallel between animals and artifacts:

Now it is in the latter of these two senses (the final cause rather than the motor cause) that either the whole soul or some part of it constitutes the nature of an animal; and inasmuch as it is the presence of the soul that enables matter to constitute the animal nature, much more than it is the presence of matter which so enables the soul, the inquirer into nature is bound on every ground to treat of the soul rather than of the matter. For though the wood of which they are made constitutes the couch and the tripod, it only does so because it is capable of receiving such and such a form. (64)a28-34)

While animal and artifact differ in the location of the final cause--the former containing it in itself, the latter receiving it from a craftsman--the operation of the form as final cause, as logos, remains the same in the artifact as in the animal. Our model for understanding the production of animals in nature remains exactly the same as the procedure for understanding the production of artifacts. The logos informs matter according to its own necessity. This necessity may vary in degree according to the sequence of the events in the particular production. Thus, human souls and animal souls alike are their own logos, their own final cause. There is less latitude in their design than in the design of a couch, but the operation of the logos remains exactly the 
same. Only the particular end or goal differs; only the site of the logos shifts: from the mind of the producer of the artifact to the soul of the animal. Within the framework of the Aristotelian metaphysics and the methodology which it demands, the logos holds a preeminent position in defining the succession of necessary events in any production.

According to Aristotle, what we know of the origin and destiny of cats is the same general notion of logos that we know in ourselves in following any procedure from restoring health to the ill, to building a house, to giving a complete description of the production of a couch. The logos defines the origin. order, means, design, and destiny. It is their principal heading. What then remains of the logos of animals that is not defined by the metaphor of human productivity?

The methodological steps Aristotle gives in this text consolidate a metaphor of productivity that can be read in two ways. On the one hand, animals are like human artifacts in that their origin is comprehended in terms of the idea that guides the production: the order of production is understood in terms of the best means for execuling the production; and the best meanis is detailed in terms of the guiding idea, the logos. On the other hand, animals are like human producers in that the final cause, the logos, resides internally, within the animals themselves. Animals also have a soul, and though the animal's soul is mortal instead of immortal and characterized by an intellect that is passive instead of active, it is its own logos as much as a human is its own final cause.

The metaphor of productivity so dominates Aristotle's conception of the logos in this analysis that there is barely any difference between the human production of an artifact and the origin and composition of animals. A cat is like a couch; we explain the origin and destiny of cats with the same procedure we use to explain the origin of furniture. Animals are simultaneously products and like the humans who produce them, and in both comparisons there is no longer a relationship of simple analogy, It is an identity. Animals are identical to artifacts because they are produced by the guiding idea or logos: they are identical to the artisan in that they contain the guiding idea themselves.
The dual effects of the metaphor of productivity in Aristotle's analysis brings with it a residue of issues which still remain to be decided 2300 years later. While he leans towards placing animals with artifacts, the margin of difference separating animals from either us or our artifacts remains unclear. On the one hand, Aristotle can state that animals are not like producers because they are products, and on the other hand he can state they are not artifactual because they produce themselves. In delineating such differences each point of comparison is the alibi for the other, and the metaphor persists in emphasizing the identities among its several terms. This lack of resolution in Aristotle's treatment comes to the fore when the ethical treatment of animals demands a decision between animals interpreted as products or producers.

With this reading of Aristotle, a new question emerges that we might pair with Thoreau's question. Do Aristotle's methodological considerations in De Partibus Animalium help us to fashion a case for the ethical treatment of animals? or do these considerations hinder us by making animals too much like ourselves and our products? In the intervening history of philosophy and culture what remains of the Aristotelian notion of the logos which is not dominated by the metaphor of productivity? Are production and the question of origin still linked in terms of some form of a guiding idea?

I will now demonstrate that the traces of this metaphor of production can be found 2300 years later in the Dasein-analytic of Heidegger's Sein und Zeit. In the passages where Heidegger turns to consider animals, we find ourselves immediately in the midst of a description of production:

But the work to be produced is not merely usable for something. The production itself is a using of something for something. In the work there is also a reference or assignment to 'materials': the work is dependent on leather, thread, needles, and the like. Leather, moreover, is produced from hides. These are taken from animals, which someone else has raised. Animals also occur within the world without having been raised at all; and, in a way, these 
entities still produce themselves even when they have been raised. So in the environment certain entities become accessible which are always ready-to-hand, but which, in themselves, do not need to be produced. $(H 70,100)^{3}$

In this text, Heidegger's phenomenological interpretation approaches animals through the roles they take on in those projects for the sake of which we labor. Our production is dependent on entities other than ourselves: some of which we produce, some which we do not produce. Heidegger then points out that animals "in a way" produce themselves. Animals are products which produce themselves. Before pursuing this striking statement, we should locate such "products" within Heidegger's analysis.

In Heidegger's methodological terminology these entities are called "ready-to-hand" (zuhanden) in the world, and their being as ready-to-hand is the mode of our production's "discovery" of them:

Our concernful absorption in whatever work-worid lies closest to us, has a function of discovering; and it is essential to this function that, depending upon the way in which we are absorbed, those entities within-the-world which are brought along in the work and with it (that is to say. in the assignments or references which are constitutive for it) remain discoverable in varying degrees of explicitness and with a varying circumspective penetration. $\langle H>1$, 101)

"The kind of Being which belongs to these entities (remember we are talking about animals along with other beings) is readiness-to-hand," and "Readiness-to-hand is the way in which entities as they are 'in themselves' are defined ontologicocatecorially." (lbid.) The being of animals is disclosed in human productivity. Our productive activity, defined by that for the sake of which we produce, shows or exhibits animals as they are in themselves.

Phenomenologically, how is this possible?
Moving backwards through Heidegger's methodological steps, I will show that Heidegger's analysis goes inside Aristotle's description of production and generation to attempt an advance beyond Aristotle by demonstrating phenomenologically a tie between the logos and the exhibition of things in their being.

Heidegger first places his own descriptive methodology in juxtaposition to what he takes as the Greeks' hermeneutic standpoint:

The Greeks had an appropriate term for 'Things': roarnuata--that is to say, that which one has to do within one's concernful dealings (npốfis). But ontologically, the specifically 'pragmatic' character of the mpámuata is just what the Greeks left in obscurity; they thought of these 'proximally' as 'mere Things'. (H68, 9697)

To Heidegger, Aristotle is not just any Greek, and so Aristotle's analysis goes one step beyond this pragmatic standpoint. It describes the necessity of the design in all beings: humans, animals, and artifacts. Aristotle recognized that methodology must be geared towards displaying this necessity 50 that the formal character of the being, the logos may be described as the guiding theme. This description exhibits the character of the logos in a two-fold way: as both discourse and the internal determination of the being. Heidegger argues that Aristotle's goal is to blend the two:

$\Lambda$ oyos as "discourse" means rather the same as $\varphi n \lambda \hat{v} v$ : to make manifest what one is 'talking about' in one's discourse. Aristotle has explicated this function of discourse more precisely as

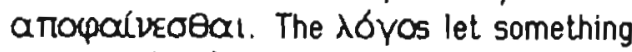
be seen ( $\varphi$ al $(v \in \sigma Q \alpha 1)$, namely what the discourse is about; and it does so either for the one who is doing the talking (the medium) or for persons who are talking with one another, as the case may be. (H32, 56) 
Heidegger's reading of Aristotle is, of course, informed by his vision of phenomenological methodology. For Heidegger, logos, in both an Aristotelian and a phenomenological description, is letting something be seen. Logos is thus at once a medium, in the sense that it conveys what is manifest, and an original act, in the sense that as apophansis--the "letting be seen"--it takes entities out of their unhiddenness, showing them in their truth, aletheia. The Being of entities within the world is revealed in itself when these entities are taken up in the "in-order-to" and the "for the sake of which" of our concernful involvements. Heidegger's Dasein-analytic purports to reveal the ontological structure of Dasein's Being in the world in terms of the apophantical "as"; it lets Dasein be seen as it is in itself. Simultaneously, this description gives us access to the other entities with which Dasein is concerned, and lets these entities be seen as they are in themselves.

We thus get a description strikingly like Aristotle's analysis of production: "Entities still produce themselves even when they have been raised. So in the environment certain entities become accessible which are always ready-to-hand, but which, in themselves, do not need to be produced." ( $(H 70,100)$ Aristotle defines both the production of artifacts and animals' own reproduction by the final cause: that for the sake of which a productive series is constituted. Heidegger defines our concernful absorption in whatever workworld lies closest to us by that for the sake of which the work is done. This purposeful intention towards entities purports to serve the function of discovering entities ready-to-hand as they are in themselves. Heidegger's analysis of production, combined with his insights into the character of the logos, claims to be a development of and a movement beyond Aristotle and by inference a mode of access to the being of animals as they are in themselves.

Given Heidegger's analysis, how might he provide an answer to Thoreau's question? In this analysis what provents animals from becoming 'mere things' that (re)produce themselves which we subsequently take up as equipment in human production? Would Heidegger also use the same procedure for describing a cat as he would for a couch? Is there any dimension of the logos as it comes into contact with the issue of animals that is independent of the motif of production?

What I take to be Heidegger's answer to these questions might not immediately satisfy us: our primary access to animals, as well as the rest of nature, is as equipment that (re)produces itself. From my reading of Aristotle's description as operating within the constraints of the metaphor of production, we might even revert to his formulation that animals are artifacts that are their own final and efficient cause. Animals are equipment with a soul. That is their logos. Heidegger could then be interpreted as adding the point that the very fact of the readiness-to-hand as equipment indicates that it has "this 'Being-in-itself' ...in which it manifests itself in its own right." (H69, 98) But what keeps this claim from simply being a reclamation and legitimation of Aristotle's description?

Heidegger separates himself from Aristotle's notion of logos as final cause in two principal ways. First, Heidegger writes:

When considered philosophically, the $\lambda 6$ yos
itself is an entity, and according to the
orientation of ancient ontology, it is
something present-at-hand. (H159, 201)

As Heidegger reads him, Aristotle is undertaking a biological-ontical exploration of life which itself relles on or is founded upon an existential interpretation of Dasein as human. Thus, the ways in which entities reveal themselves as ready-to-hand is ontologically prior to the disclosures of entities as present-at-hand. In the latter mode, we take entities as objects of scientific investigation. In the ontological analysis of the ready-to-hand, our access to these entities is through our experience of Being, working, and dying in the world. Any biological report which gives information about "living" reflects the fact that Dasein lives and experiences "life" existentially. Logos is thus tied to a human experience of existence, and is not an entity in its own right.

Secondly, Heidegger, at least the Heidegger of Sein und Zeit, criticizes Aristotle for not seeing that the only mode of access upon which a description of 
beings can be based is Dasein's experience of Being. This experience forms the phenomenological context and the hermeneutic standpoint of the Daseinanalytic:

Aristotle did not pursue the analytical question as far as the problem of which phenomenon within the structure of the $\lambda$ byos is the one that permits and indeed obliges us to characterize every statement as synthesis and dieresis. In accordance with this structure, something is understood with regard to something: it is taken together with it, yet in such a way that this confrontation which understands will at the same time take apart what has been taken together, and will do so by articulating it interoretatively. If the phenomenon of the 'as' remains covered up, and above all, if its existential source in the hermeneutical 'as' is veiled, then Aristotle's phenomenological approach to the analysis of the $\lambda 6$ yos collapses to a superficial 'theory of judgment,' in which judgment becomes the binding or separating of representations or concepts. (H159, 202)

Because Aristotle did not analyze the phenomenon of $\underline{\log 05}$ as a letting something be seen apophantically. as a letting something be seen as $\mathrm{It}$ is manifest in itself, he failed to see logos as the medium by which the disclosure of entities takes place. He instead identified it as the final cause of the entity, as the for-the-sake-of-which of the entity, as an entity in its own right.

As I read Heidegger, the difference between his phenomenological description and Aristotle's analysis is that Heidegger focuses on the locus of the description in Dasein's experience, and he takes that locus as the phenomenological point of access to the phenomenon to be described. Heidegger answers the question: "How do we legitimately obtain the phenomena to be described?"

For Heidegger what remains to be done is to work out the question of what it means to attain the being of an entity. When we comport ourselves towards beings in terms of our own productive activity, we find logos to be the medium by which the disclosure of being takes place. The logos is no longer the revealed being as it was in Aristotle; it is now the medium of revelation. For Heidegger, questions about logos remain within the Soinsfrage and within the issue of identity posed by the grammar of the copula. They remain questions which philosophy has yet to take up in terms of their full significance. Of these issues Heidegger writes:

How far this problematic has worked its way into the interpretation of the $\lambda \delta$ yos, and how far on the other hand the concept of 'judgment' has (by a remarkable counterthrust) worked its way into the ontological problematic, is shown by the phenomenon of the copula. When we consider this 'bond,' it becomes clear that proximally the synthesis-structure is regarded as seifevident, and that it has also retained the function of serving as a standard for interpretation. But if the formal characteristics of 'relating' and 'binding' can contribute nothing phenomenally towards the structural analysis of the $\lambda$ oyos as subject matter, then in the long run the phenomenon to which we allude by the term 'copula' has nothing to do with a bond or binding. The Interpretation of the 'is,' whether it be expressed in its own right in the language or indicated in the verbal ending, leads us therefore into the context of problems belonging to the existential analytic, if assertion and the understanding of Being are existential possibilities for the Being of Dasein itself. When we come to work out the question of Being (cf. Part I, Division 3), we shall thus encounter again this peculiar phenomenon of Being which we meet within the $\lambda 6$ yos. (H16, 202-203)

In this text Heidegger defers to the later-abandoned third part of Sein und Zeit on precisely the issue that concerns us here. To what extent is an animal its logos, the final cause in its production? To what 
extent do human acts of production grasp the logos of animals as either medium or entity? In answer to these questions, I think that Heidegger's analytic only improves over Aristotle's in that it straightforwardly acknowledges that our only access to the being of animals, to knowledge of their origin and destiny, is through the medium of our purposes, our for-the-sake-of-which.

Thus I hald that Heidegger, too, uses the metaphor of productivity as an alibi for avoiding a discussion of what remains of the logos outside of human activities. What Heidegger calls Aristotle's phenomenological description attains the being of animals with the same procedure we would use if we were to glve a description of a couch: the procedure we would use for any artifacl, any piece of equipment. Heidegger only modifies Aristotle's approach slightly by pointing out that our mode of access to entilies in the world is through our intentions and projects. We attain the being of animals as they are in themselves by disclosing them as equipment. Logos, whether in Aristotle or in Heidegger, remains a distinctly human phenomenon; it remains a metaphor of design imbedded in the human activity of production.

or course the question arises, "Does a logos for animals remain which is not defined by our species or attained for reflection through the purposes of our species?" Thoreau's challenge to us as philosophers thus remains. Do cats have an origin and destiny outside the human domain of meaning, purposes, procedures, and productivity? Every cat owner, of course, has the suspicion they do.

In Heidegger's text, such questions are acknowledged, but only as an unfinished remainder of the Dasein-analytic. Heidegger states that it would be interesting to raise the question of the Dasein of animals, but this is a very difficult matter. He even closes the subject of animals by asking, "It remains a problem in itself to define ontologically the way in which the senses can be stimulated or touched in something that merely has life, and how and where the Being of animals, for instance, is constituted by some kind of "time'." (lbid.) This quote is reminiscent of the lack of resolution that 1 found in Aristotle. Again it is appropriate to ask if there is a margin of difference that separates animals from either us or our products? Much like the problems the phenomenon of $\tau$ to yos implies for the existential analytic, these questions about animals are a supplement to the text that was never written, literally an untouched remainder of the logos.

The dominance of the vocabulary of production guided by logos in Aristotle's and Heidegger's discussions of the being of animals makes Thoreau's question all the more a spur to our philosophizing. In response to Thoreau's question we should ask, "What kind of philosophy is it that asks questions about the origin and destiny of cats as a way of measuring the success of philosophy?" The answer ! have found is that it is a philosophy that searches for reasons and causes in being itself, to the end of discovering the origin of beings. It is a philosophy that searches for the logos. This search, in the texts I have taken up here, takes place within the framework of the guiding motif of production.

When we push the issue further and ask what remains of the logos--whether understood as entity or medium--of animals which is not dominated by the motif of production, we find in both Aristotle and Heidegger that the being of animals is constituted as a lack. In the passage just quoted from Heidegger, animals are explicitly described as "something that merely has life." May we also presume then that animals have no projects or acts of production for the sake of which their actions are done? Heidegger would certainly say. "Not in an existential or historical sense." Looking at the same issue in Aristotle we find that while animals have a soul which allows them to be their own final cause, it is a soul which lacks an agent intellect. Again we find that the logos of animals is defined in terms of lack.

What remains of the logos which is not governed by the metaphor of production? The answer I find in the texts of Aristotle and the early Heidegger is that this remainder is not lacking but is itself conceived in terms of a lack. This lack is doubly unresolved: animals lack the requisites to be either human producers or to be pure products. We neither produce them nor do they produce, and all the while we attempt to comprehend them within this paradigm.

I would like to close with a provisional reflection on the import of these observations for the study of 
ethics and animals. Both the meaning of logos in the Aristotelian notion of the source of the productive design that is common to both human and animal souls and the Heideggerian description of the medium by which the design is exhibited place the logic of arguments that seek ethical clarification about animais in a double-bind. Most, if not all, inquiries about ethics and animals remain within the understanding determined by the metaphor of production. While we attempt to investigate issues of the morality and immorality of our behavior towards animals as means to our productive ends. we simultaneously strain to escape the gravitational pull of the metaphor of production and establish an ethics for animais outside of human activity. This argument transcends the standpoints of utilitarian and deontological views because ethics is always the consideration of the behavior of moral agents, and moral agents are always productive agents. We can of course ask. "Are the human ends which subsume animals as means moral or immoral?" But when we ask. "Are the actions of animals--whether raised in captivity or found in nature--subject to standards of justice?" only our question remains.

The study of ethics and animals, then, seems to become a matter of both judging our own actions towards animals and exempting animals from the standards for our behavior. When we doubt the morality of making animals the means to our productive ends, we have to exempt animals from the obligations of an autonomous and rational moral agent as we simultaneously grant them that same status. This paradox is the manifestation in ethics of the same unresolved double-bind within which we found Aristotle and Heidegger when they concluded that animals are products which produce themselves. We transfer to animals our own privileged status of an end-in-itself without granting that animals are capable of moral action. Here, too, we find a lack: animals lack the capacity for moral obligations. The result is that we share half our human status with them: animals have a moral status but without the simultaneous acknowledgment that the ends or goals of animals are subject to moral imperatives.

Thoreau himself might have attempted an argument that would guide us out of this ethical bind. Starting from the premise that "All good things are wild and free," we could, by an action of sympathetic imagination, will for animals the metaphor which conditions our understanding of ourselves: the capacity to produce and the individual determination of origin and destiny. Along this line we could argue that animals are, like us, capable of producing themselves. Not knowing the origin and destiny of cats, philosophical speculation can at least posit the condition for there to be an origin and destiny independent of human ends. We will not grant that animals are capable of moral action, but we will grant them the rights of independent moral agents. Still, they continue to be taken up as means to our ends.

The ethical consideration of the treatment of animais disintegrates as it is pushed towards its ground. Ethical arguments determine the status of animals within the framework of a metaphor of productivity. The protection of animals in sanctuaries and preserves illustrates the double-bind of this logic. We establish "kingdoms" or preserves or laws intended for some few of them to pursue their own ends. Doing this for some, however, is not the same as doing the same for all, and the perceived interests of a productive society prohibit the categorical application of the protectionist's standpoint. Even if we match our own origins to that of animals in a modification of Darwin's hypothesis of a planet-wide evolutionary system, this hypothesis only aggravates Thoreau's question by first establishing common conception of an evolutionary ground which we have already determined as our mutual origin and, second, by deeming the question of destiny irrelevant in light of natural selection and historical accident. Thoreau's question both cites and is situated in an unaddressed remainder in our philosophical tasks: to question the metaphor of the productive agent that guides our thinking, to say nothing of the origin and destiny of cats.

\section{NOTES}

1. Aristotle, The Parts of Animals, translated by A.L. Peck, with a foreword by F.H.A. Martin (Cambridge: Harvard Universily Press, 1937). 
2. Aristotle, The Works of Aristolle, translated in English under the editorship of J.A. Smith and W.D. Ross, Vol. $\vee$ De Partibus Animalium (Oxford: The Clarendon Press, 1912). All subsequent references to Aristotle are from this edition.

3. Martin Heidegger, Being and Time, translated by John Macquarrie and Edward Robinson (New York: Harper and Row, 1962).

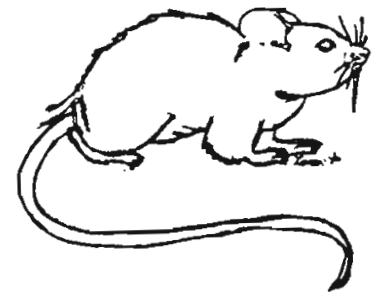

\section{BOOKS RECEIVED}

\section{J. Ronald Engel \& Joan Gibb Engel}

ETHICS OF ENVIRONMENT AND DEVELOPMENT

Global Challenge, International Response

Tucson: The University of Arizona Press, 1990

Foreward, preface, 253p, index

$\$ 29.95$ clothbound

Michael W. Fox

ST. FRANCIS OF ASSISI, ANIMALS, AND NATURE Washington: The Humane Society of the United

States, 1989

$35 p$

$\$$ ? paper

Kathleen Malloy

WILD HEART

St. John, Kansas: Kindred Spirit Press, 1990

Foreward, 29p

$\$$ ? paper

Clive Roots

THE BAMBOO BEARS

The Life and Troubled Times of the Giant Panda Winnipeg: Hyperion Press Limited, 1989

$99 p$, selected bibliography, index

$\$ 17.95$ paperback

\section{OTLUIER RATTOCR}

The sovereign nations of the birds

Pursue their own affairs.

What I consider my domain

They look upon as theirs.

Their kingdoms are as great as mine.

They want no mis-alliance.

They reckon not my suzerainty

But sing in wild dafiance.

\section{- - Gary KowalskI}

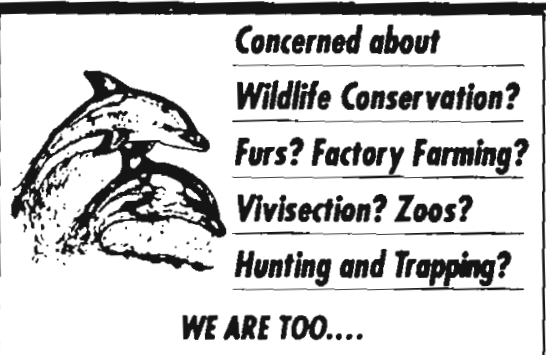

Did you know thot philosophers have also mode a contribution to the growth of the onimol liberation movement? - Think of Regan, Singer, Clork, Mogel, Rollin and Sopontis.

Botwonen the Spadies "is the only publication which allows surh exiensive examination of the philosophical bosis for onimal rights." - Rrod Mthlor, Humone forming Association

Subsartbe today - and please send your tox deductible contribution -help us guarantee philosophers a forum in which to continue to evolve a sound basis for onimol rights.

$\$ 15.00$ from

Son Froncisco Boy Instifule

P.O. Box 254

Berkeley, CA 94701

Somple bock issue $\$ 3.00$

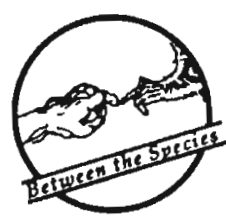

Quarterly Joumal of Ethics

\section{PHILOSOPHICAL ACTIVISM} NEEDS YOUR SUPPORT! 